\title{
Preparation of Silica Films on Ru(0001): a LEEM/PEEM Study
}

\author{
H.W. Klemm, G. Peschel, E. Madej, A. Fuhrich, M. Timm, D. Menzel, Th. Schmidt, and H.-J. Freund \\ Department of Chemical Physics, Fritz-Haber-Institute of the Max-Planck Society, Faradayweg 4-6, 14195 Berlin, Germany
}

\begin{abstract}
We use an aberration corrected spectro-microscope, the low energy electron microscope/photoelectron emission microscope (LEEM/PEEM) SMART, to follow the preparation and structure of a bilayer silica film on $\mathrm{Ru}(0001)$ as function of temperature and oxidation conditions. This allows us to analyze the growth process at different length scales in order to judge on the overall quality and the morphology of the film. It is found that the film growth occurs in a crystalline and a vitreous phase as previously discovered using scanning tunneling microscopy. However, the present experiment allows an analysis on the sub-micron level to gain insight into the growth process at a mesoscopic scale. We find that the fully oxidized film can be prepared but that this film contains holes. These are unavoidable and are important to consider, if one wants to use the films for ensemble averaging experiments to investigate migration and reaction of molecules between the silica film and the $\mathrm{Ru}(0001)$ substrate.
\end{abstract}

Keywords: silica, oxide films, growth, LEEM, XPEEM, LEED, XPS

\section{Introduction}

The most abundant minerals on the earth's crust are silicates. The mother compound, of course, is quartz and it comes as crystalline and vitreous or glassy phases.[1] The discovery of the details of the vitreous-crystal transition is still to come, and we and collaborators have recently made a step towards unraveling the structure of both, the crystalline as well as the vitreous phase in real space by scanning tunneling (STM) and atomic force microscopy (AFM) of a bilayer silica film grown on $\mathrm{Ru}(0001)$ as shown in Fig.1.[2-13] One can clearly identify the silica ring structure composed of $\mathrm{SiO}_{4}$-tetraedra connected into a hexagonal network of rings in the crystalline phase, while in the vitreous phase the connectivity changes and allows for larger and smaller ring systems, as predicted 80 years ago by Zachariasen.[14] After our discovery similar films have been prepared and characterized on graphene [15, 16], Pd (100) [17] and Pt(111) [18] using STM and transmission electron microscopy. However, those characterization techniques usually do not allow a sufficiently wide scanning range so that conclusions can only be drawn on small areas of silica covered Ru surfaces. On the other hand, if one is interested in properties of such systems, which have to be investigated using ensemble-averaging techniques, this requires information on at least a mesoscopic length scale in order to draw representative conclusions. For example, we are interested in the investigation of diffusion of molecules of different sizes through the silica film in order to study chemistry in constrained space, a topic that came up first with zeolites [19], but has now been 
also applied to diffusion and reaction under metal supported graphene sheets.[20-24] Graphene on the other hand is considerably more strongly coupled to the metal than the van-der-Waals bound silica bilayers. Therefore, for the latter one expects a less pronounced influence of the covering layer beyond representing a membrane for diffusion of species and a means to constrain the space. We have undertaken first attempts to study diffusion underneath the silica film using infra-red spectroscopy.[25] For such studies it is essential to know the structure and morphology of the bilayer on a considerably larger, mesoscopic length scale, because holes in the layer would influence diffusion underneath the layer considerably, and would lead to false conclusions if interpreted at a microscopic level. In the present study, we follow the preparation of a bilayer silica film at the mesoscopic level using low energy electron microscopy in conjunction with photoelectron emission spectroscopy (LEEM/PEEM), using synchrotron radiation.[26-29] Ultimately, this would allow to study the distribution of crystalline and vitreous phases across the surface and to investigate changes as a function of temperature. In conjunction with atomically resolved scanning probe techniques, this would bring us closer to a detailed understanding of the vitreous-crystal phase transition. In this paper, we present the first steps towards this goal by investigating the preparation conditions for crystalline and vitreous films at the mesoscopic scale.

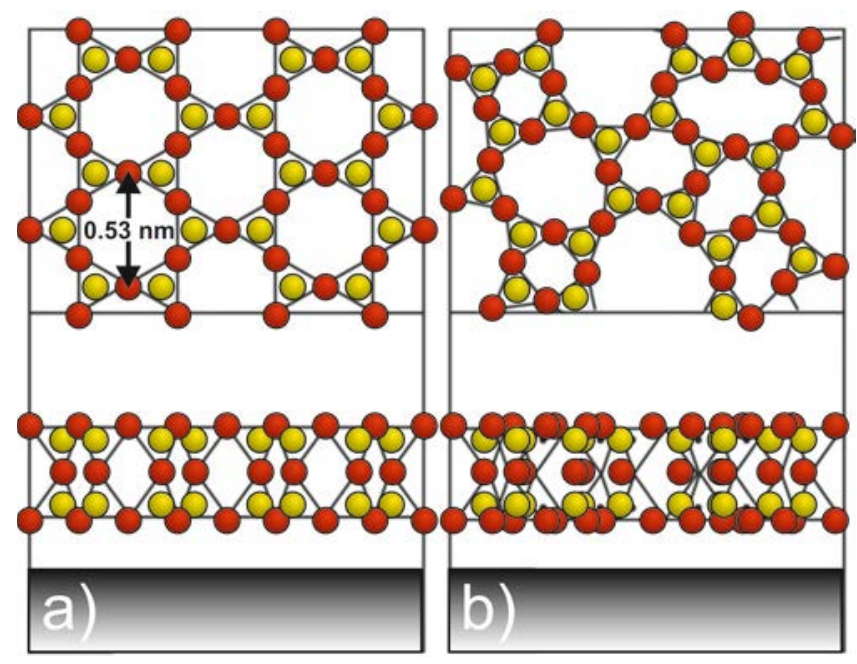

Fig. 1. Model of crystalline and vitreous phases of a silica bilayer, Si and O atoms in red and yellow, respectively. .

\section{Experimental}

The experiments were carried out in the SMART microscope operating at the UE49-PGM beam line of the synchrotron light source BESSY II of the Helmholtz Center Berlin (HZB). This aberration corrected and energy filtered LEEM/PEEM instrument combines microscopy, diffraction, and spectroscopy techniques for comprehensive characterization of surfaces. The base pressure of the system is $10^{-10} \mathrm{mbar}$; however, operation at oxygen pressure of up to $10^{-5} \mathrm{mbar}$ and at temperature above $1300 \mathrm{~K}$ is possible.

The $\mathrm{Ru}(0001)$ single crystal had a miscut below $0.2^{\circ}$ and was pretreated by cycles of (i) argon sputtering for $30 \mathrm{~min}$ at room temperature $(\mathrm{RT})\left(\mathrm{U}_{\text {bomb }}=1500 \mathrm{~V}, \mathrm{I}_{\mathrm{s}}=5 \mu \mathrm{A}\right)$, (ii) oxygen treatment for $5 \mathrm{~min}$ at $1170 \mathrm{~K}$ 
at $p_{02}=1 \times 10^{-6}$ mbar and (iii) annealing in UHV for 5 min at $1420 \mathrm{~K}$ and subsequently for $1 \mathrm{~min}$ at 1520 $K$. This cleaning was repeated until no contamination could be detected by XPS and the Ru(0001) surface showed a few $100 \mathrm{~nm}$ wide terraces with straight step edges (to be seen as black lines in Fig 2a and b), and a sharp and bright (1x1) LEED pattern appeared (see Fig 2e). The sample temperature was measured either by a W26\%Re/W5\%Re thermocouple, spot-welded to the crystal support, or by a pyrometer (Lumascence Technologies, IMPAC IGA 740) with an absolute accuracy of about $10 \mathrm{~K}$. Oxygen (99.999\% purity) was dosed by a leak valve directly into the main chamber; silicon was sublimated from a $4 \mathrm{~mm}$ thick rod (99.999\% purity, Matthias Schmehl Laborausstattung, Rostock) using a commercial evaporator (Omicron EFM3 with ion suppressor) pointing towards the sample under grazing incidence of $20^{\circ}$. The deposition rate of the evaporator was calibrated in two ways: first silicon was grown on a Si(111) surface, where the layer-by-layer growth could be directly observed. Here, the different atom density for $\mathrm{Si}(111)$ and silica layer had to be taken into account. One monolayer (ML) of silica is defined as a closed layer of $\mathrm{SiO}_{2}$ on $\mathrm{Ru}(0001)$. A second way of calibration evaluated the XPS intensities ratio for the two oxygen peaks, related to bonding to silicon and to ruthenium, respectively.

\section{Results and Discussion}

Two different preparation recipes were applied to study the influence of the various parameters and the properties of mesoscopic defects. Whereas in the so-called "standard recipe" the Si deposition and the oxidation are done in separate steps, the silicon is oxidized during deposition in the second recipe, i.e. reactive deposition.

\subsection{Preparation of silica film: "Standard recipe"}

The common recipe for the preparation of silica as described in Ref. [8] consists of three subsequent steps: (i) preparation of the $\mathrm{Ru}(0001)-\mathrm{p}(2 \times 2)-30$ layer, (ii) $\mathrm{Si}$ deposition at RT and low oxygen pressure, and finally (iii) oxidation. The (2x2)-30 surface is produced by keeping the Ru substrate in $1 \times 10^{-6} \mathrm{mbar}$ of oxygen at $1170 \mathrm{~K}$ for $10 \mathrm{~min}$. The oxygen pressure is not reduced before cooling down below $470 \mathrm{~K}$. Compared to the initial Ru(0001) surface (Fig. 2a and e) the step density and shape do not change (Fig. $2 \mathrm{~b})$, but the LEED pattern switches to a $\mathrm{p}(2 \times 2)$ reconstruction with sharp spots (Fig. 2f). As clearly seen in dark field imaging, this structure has threefold symmetry, rotated by $180^{\circ}$ between neighbouring surface terraces due to the $A B A B$ stacking of the $R u(0001)$ substrate with hcp crystal structure. However, there are no rotational domains within the oxygen layer on one substrate terrace. In a second step, $2 \mathrm{ML}$ of silicon were deposited at RT in $2 \times 10^{-7}$ mbar of oxygen, resulting in a rough surface with objects of 50 to $100 \mathrm{~nm}$ in size, as seen in mirror microscopy MEM (Fig. 2c), without structural order (no spots in LEED, Fig. 2g). During the deposition, the intensity of the LEED spots and of the LEEM image decreased exponentially. For the final oxidation step, the film was heated up in $5 \times 10^{-6}$ mbar of oxygen with a rate of $1-5 \mathrm{~K} / \mathrm{s}$. The annealing temperature of $1045 \mathrm{~K}$ was kept for $10 \mathrm{~min}$, until the sample was cooled down with $1 \mathrm{~K} / \mathrm{s}$ in oxygen pressure, which was not reduced before $470 \mathrm{~K}$ was reached. The surface appears still rough (Fig. 2 d), but compared to Fig. $2 c$ the domains are clearly smaller with 20 to $50 \mathrm{~nm}$ in size, and substrate atomic steps and step bunches are again visible. The silica film exhibits also 
a $\mathrm{p}(2 \times 2)$ LEED pattern (Fig. $2 \mathrm{~h}$ ); compared to the 30-(2x2) pattern (Fig. $2 \mathrm{f}$ ) the half order spots are more intense but less sharp, indicating a structural change within the unit cell and smaller domain size. Differing from the $30-(2 \times 2)$ surface, no contrast was found in dark field imaging using the $p(2 \times 2)$ superstructure spots.

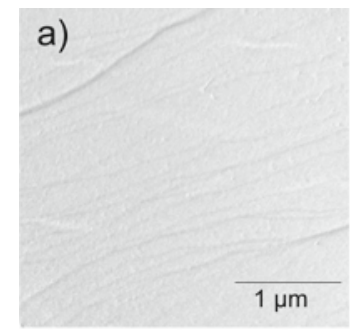

e)

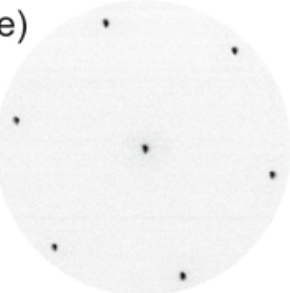

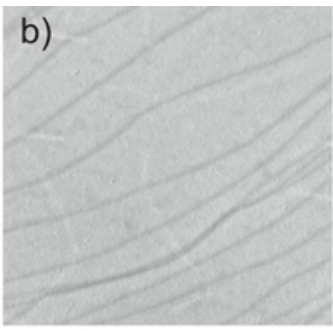

f)

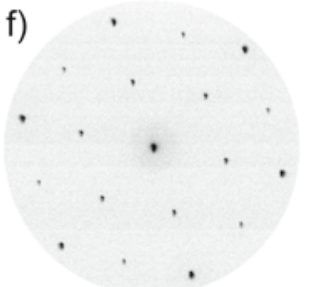

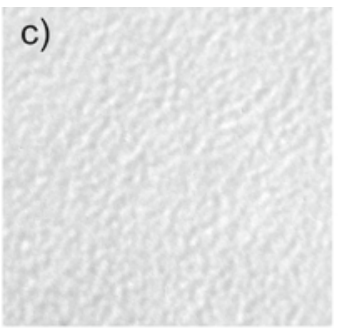

g)

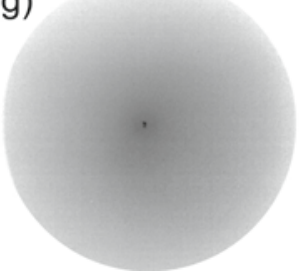

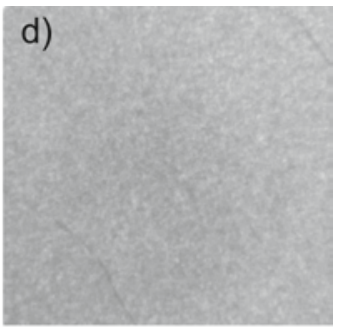

h)

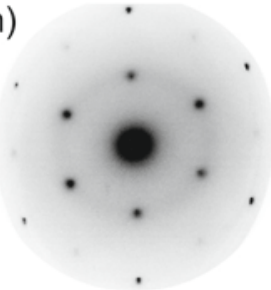

Fig. 2: Stepwise preparation of silica film on $\mathrm{Ru}(0001)$ surface. LEEM images (top row) and corresponding LEED patterns (bottom) of clean $\mathrm{Ru}(0001)$ surface ( $a$ and $e), \mathrm{Ru}(0001)-(2 \times 2)-30$ (b and f), Si deposited in $\mathrm{O}_{2}\left(\mathrm{p}_{\mathrm{O} 2}=2 \times 10^{-7} \mathrm{mbar}\right.$ ) ( $\mathrm{c}$ and $\left.\mathrm{g}\right)$, and Si film annealed in $\mathrm{O}_{2}\left(\mathrm{p}_{\mathrm{O} 2}=5 \times 10^{-6} \mathrm{mbar}, \mathrm{t}=10 \mathrm{~min}\right.$, $1045 \mathrm{~K})\left(\mathrm{d}\right.$ and $\mathrm{h}$ ). All LEED patterns were taken at $\mathrm{E}_{\mathrm{kin}}=42 \mathrm{eV}$ and are shown in logarithmic scale, the LEEM images at $E_{\text {kin }}=12 \mathrm{eV}(\mathrm{a}, \mathrm{d}), 14 \mathrm{eV}(\mathrm{b})$ and $1 \mathrm{eV}(\mathrm{c})$.

\subsection{Temperature dependence during deposition in oxygen atmosphere}

The small domain size observed at the RT deposition of silicon and after the oxidation might be caused by limited diffusion during the processes. With the aim to enhance the domain size, we studied the influence of the Si deposition temperature, keeping the oxygen pressure at $2.5 \times 10^{-6}$ mbar during the growth. The process was observed in situ and in real-time by LEEM and/or LEED during Si deposition at constant temperature. This preparation was repeated for different temperatures in the range between $670 \mathrm{~K}$ and $1120 \mathrm{~K}$. Fig. 3 shows the morphology of the resulting silica layers (dark areas in the LEEM images) after deposition of about $0.7 \mathrm{ML}$. Clearly, the density of nucleated silica islands decreases with increased deposition temperature. At the same time, the island size increases and the shape becomes more fractal at higher temperature. Whereas at $670 \mathrm{~K}$ (Fig. 3a) the island size and distance are smaller than the resolution limit of $\sim 15 \mathrm{~nm}$ in this measurement, the silica islands are clearly visible above $900 \mathrm{~K}$. Besides the homogeneous nucleation on the terrace, a preferred nucleation at the substrate steps is observed at higher temperature, seen already in the early stage of growth as step decoration. The higher the temperature the more pronounced is the dendritic shape of the islands. Also, branches grow nearly perpendicular to both sides of the decorated steps. Though the deposited silicon amount is the same for Fig. 3a-e), the fraction of silica area is greatly reduced at higher temperature. Even if the growth is continued towards $1120 \mathrm{~K}$, the island size is not increasing, indicating either three-dimensional growth or desorption of the silica at that high temperatures - the latter explanation is supported by XPS 
data. The temperature dependence of the island density is shown in Fig. 3f). Here, the density is determined on the flat terrace, not influenced by the decorated substrate steps. The fit in the Arrhenius plot has a slope corresponding to an activation energy of $E_{n}=1.65(+/-0.15)$ eV. Furthermore, LEED shows always a $p(2 \times 2)$ structure, expected for both the silica film and the $30-(2 \times 2)$ layer between the islands, indicating that the silica film grows with an ordered structure. XPS at Si $2 p$ and Ru $3 d$ provide proof that no alloy phase is formed.
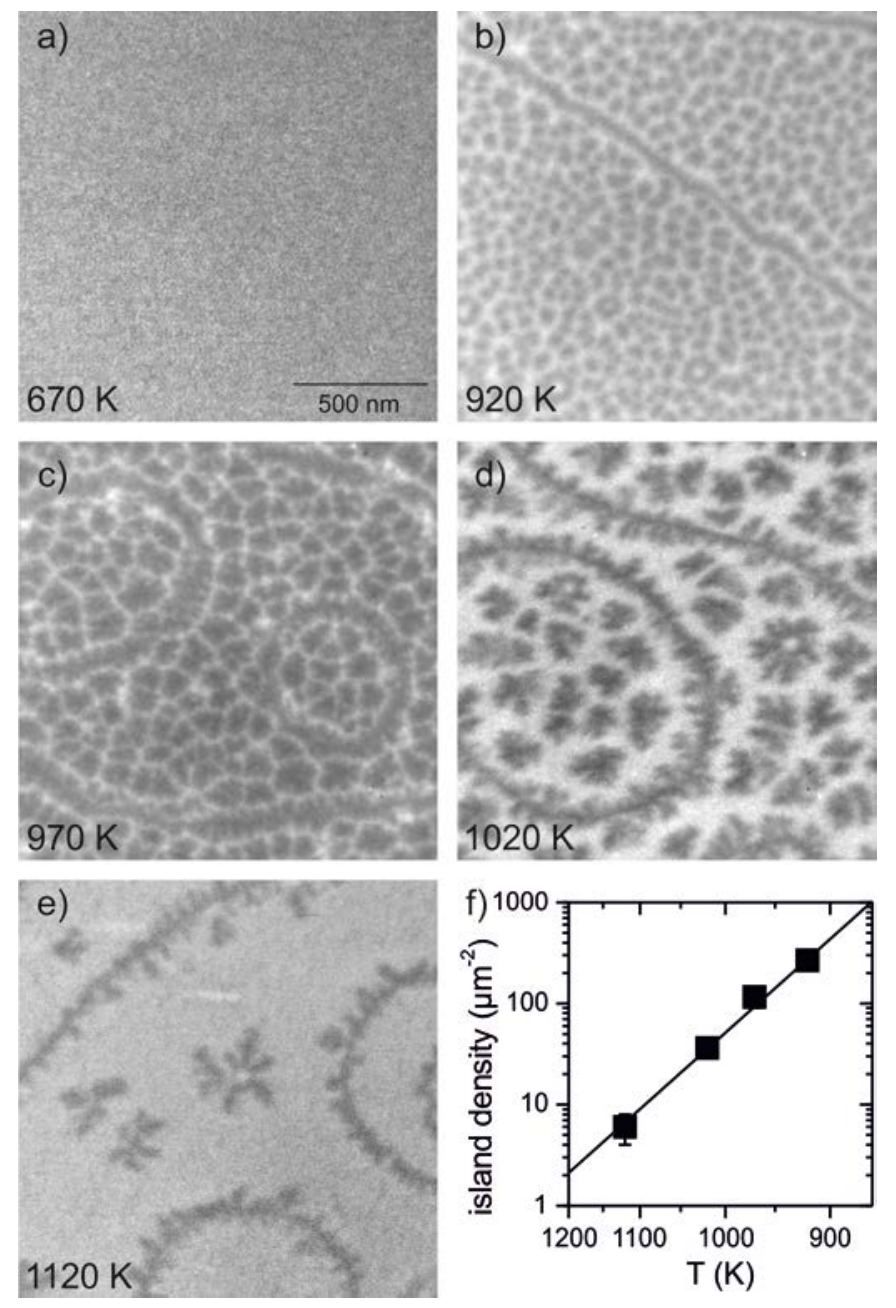

Fig. 3: LEEM images of different reactive deposition experiments. About $0.7 \mathrm{ML} \mathrm{Si}$ is deposited in $\mathrm{p}_{\mathrm{O} 2}=$ $2.5 \times 10^{-6} \mathrm{mbar}$ at temperatures as indicated. Images taken under preparation condition. Electron energy is $E_{\text {kin }}=22 \mathrm{eV}(a, b, d), E_{k i n}=11 \mathrm{eV}(c)$ and $14 \mathrm{eV}(\mathrm{e})$. The temperature dependence of island density, determined on larger terraces, is shown in $\mathrm{f}$ ).

The Ru and Si XPS lines were used for element specific XPEEM imaging (Fig. 4a and b) in comparison with the structurally sensitive LEEM images (Fig. 4c). Clearly, the islands grown at $1020 \mathrm{~K}$, and appearing dark in LEEM, can be identified as areas containing silicon (bright in Fig. 4b). This contrast is inverted for the $\mathrm{Ru}$ line (Fig. $4 \mathrm{a}$ ), because the Ru $3 \mathrm{~d}$ signal of the underlying substrate is damped by silica islands, but strong between the islands. 


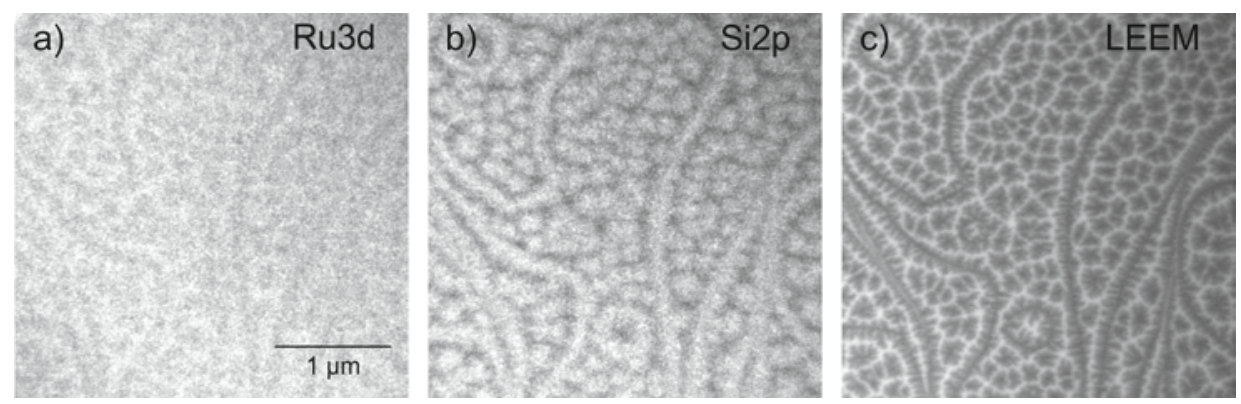

Fig. 4: XPEEM images of a) Ru 3d, hv = $354 \mathrm{eV}, E_{\text {kin }}=73 \mathrm{eV}$ and b) Si 2p, hv = $175 \mathrm{eV}, \mathrm{E}_{\mathrm{kin}}=69 \mathrm{eV}$; c) LEEM image $E_{\text {kin }}=21 \mathrm{eV}$. Silica film produced at $1020 \mathrm{~K}$ in oxygen.

\subsection{Temperature dependence during final oxidation, standard recipe}

The standard recipe, as described in section 3.1, also shows a temperature influence. Figure 5 displays LEEM (top row) and LEED (bottom) from nominally two ML thick silica films, oxidized at different temperatures. While at $995 \mathrm{~K}$ and $1045 \mathrm{~K}$ the resulting silica film is completely closed, even at the atomic steps of the substrate (dark line crossing diagonally Fig. 5a in 5b), the film oxidized at $1270 \mathrm{~K}$ is strongly de-wetted, as seen in the wide darker area on the left side of Fig. $5 \mathrm{~d}$. These dark areas always appear along substrate steps and step bunches (crossing diagonally from lower left corner to the top centre in Fig. 5). Obviously, the de-wetting starts at the substrate step where either the strain in the film is higher or the bonding within the silica rings is incomplete, triggering break-up of the structure. Furthermore, these de-wetted areas show black and dark grey patches, representing monolayer silica and holes of oxygen covered Ru substrate. The bright silica area also is inhomogeneous: it shows dark dots of about $20 \mathrm{~nm}$ size, representing most likely holes in the bilayer filled with the silica monolayer, or areas with the oxygen covered Ru substrate. Temperature effects can also be seen in the LEED pattern: whereas the silica film, produced at lower oxidation temperature, exhibits the expected $p(2 \times 2)$ structure (Fig. 5e and 5f), oxidation at higher temperature yields an additional structure, which is identified as a $(2 \times 2) R 30^{\circ}$, rotated by $30^{\circ}$ against the dominant $p(2 \times 2)$ structure and therefore is incommensurate to the $\mathrm{Ru}(0001)$ substrate. At an intermediate oxidation temperature of $1095 \mathrm{~K}$ LEEM shows white dots, about $20 \mathrm{~nm}$ in size, but no dark areas (Fig. 5c), and LEED (Fig. 5g) displays a strong $\mathrm{p}(2 \times 2)$ structure with a weak contribution of a $(2 \times 2) R 30^{\circ}$ structure. 


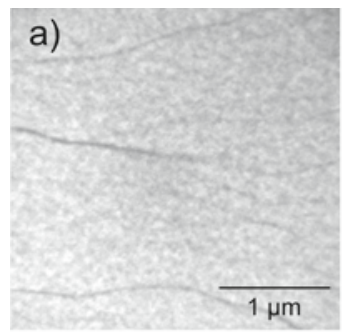

e)

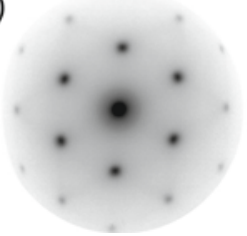

b)

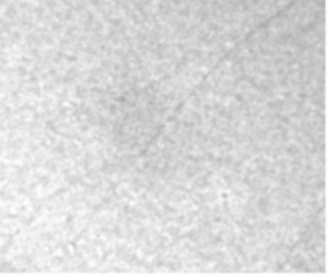

f)

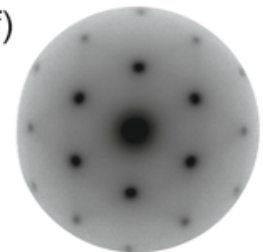

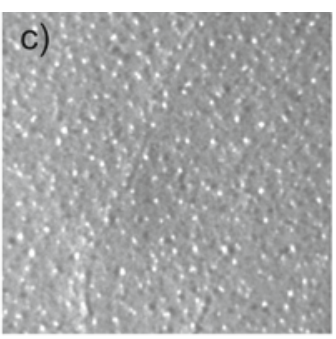

g)

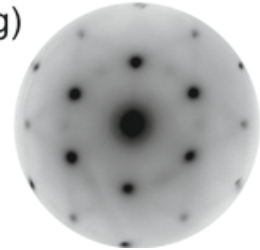

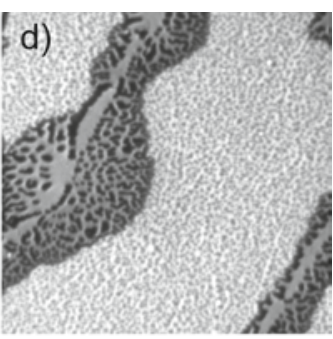

h)

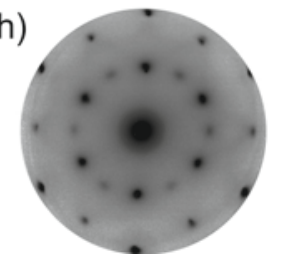

Fig. 5: LEEM images and corresponding LEED pattern after oxidation at $T=995 \mathrm{~K}(\mathrm{a}, \mathrm{e}), 1045 \mathrm{~K}(\mathrm{~b}, \mathrm{f})$, $1095 \mathrm{~K}(\mathrm{c}, \mathrm{g})$ and $\mathrm{T}=1270 \mathrm{~K}(\mathrm{~d}, \mathrm{~h})$. Kinetic energy $E_{\mathrm{kin}}=42 \mathrm{eV}$.

The origin of the white dots is revealed in Figure 6, where the same surface area is shown in LEEM (Fig. $6 \mathrm{c}$ ) and XPEEM, using the Si $2 p$ and Ru 3d XPS lines (Fig. 6a and b). In analogy to Fig. 4, the contrast in the Ru and Si-XPEEM images is inverted, showing that the small white dots in LEEM are holes in the bilayer silica film. Additionally, the influence of substrate step bunches can be seen in the upper left (bright band in LEEM, dark grey in Si-XPEEM). Clearly, at the step bunches the Si concentration is reduced, and therefore the substrate Ru signal is less damped. However, in the step bunch area the white dots in LEEM, appearing dark in Si-XPEEM and bright in RU-XPEEM, are still visible. From this we conclude that the Si amount on the step bunch area most likely corresponds to one monolayer, so that the holes reach the Ru substrate. Once these holes are formed, they cannot be filled up later, neither by deposition of additional $\mathrm{Si}$ and subsequent oxidation, nor by Si deposition at elevated temperatures in an oxygen atmosphere. This is similar to the $\mathrm{Fe}_{3} \mathrm{O}_{4} / \mathrm{Pt}(111)$ system, where holes formed by dewetting can also not be closed by additional Fe deposition and oxidation.[30] Obviously, this additionally deposited material is not incorporated in the hole area, but prefers thermodynamically to migrate upward on top of the oxide film.
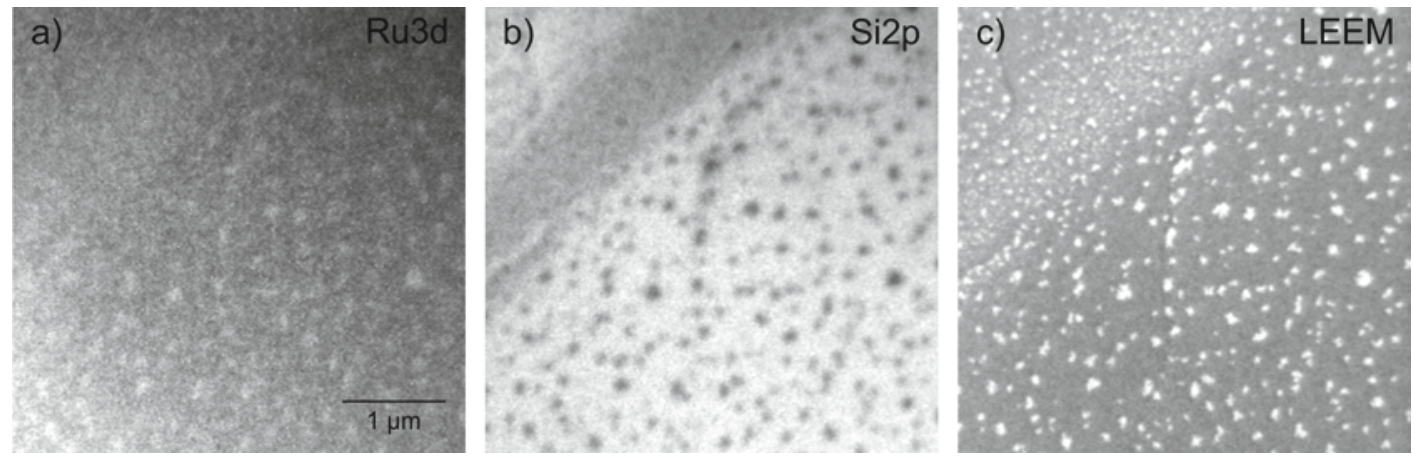

Fig. 6: Silica film after oxidation at $1095 \mathrm{~K}$. XPEEM images of a) Ru 3d, hv $=360 \mathrm{eV}, \mathrm{E}_{\mathrm{kin}}=79 \mathrm{eV}$ and b) Si $2 p, h v=200 \mathrm{eV}, E_{\text {kin }}=95 \mathrm{eV}$; c) LEEM image at $E_{\text {kin }}=79 \mathrm{eV}$ 
Up to now, we focussed on the effect of oxidation temperature on the morphology of the silica film. The second influence is the degree of oxidation which we investigated by XPS using the Si $2 p$ and O 1s lines. The binding energy of the Si $2 p$ line shifts strongly with the oxidation state [31]: whereas silicon shows the $2 p$ state at $E_{B E}=99.3 \mathrm{eV},[32]$ the fully oxidized $\mathrm{Si}^{4+}$ state is known to be shifted towards higher binding energy by about $4 \mathrm{eV}$. This can also be seen in Fig. 7a) for the oxidation at $995 \mathrm{~K}$ (black line), where the Si $2 p$ can be deconvoluted into these two components. Obviously, the film consists of areas with completely oxidized $\mathrm{Si}\left(\mathrm{E}_{\mathrm{BE}}=103.3 \mathrm{eV}\right)$ and of areas with only partially oxidized or even not oxidized silicon (between $\mathrm{E}_{\mathrm{BE}}=99.3 \mathrm{eV}$ and 101.3eV). At higher oxidation temperature (red and blue curves), the Si $2 p$ does not show the peak at $\mathrm{E}_{\mathrm{BE}}=99.3 \mathrm{eV}$, indicating complete oxidation. However, the peak is shifted towards lower binding energy by $0.6 \mathrm{eV}$ and $1 \mathrm{eV}$ at $1045 \mathrm{~K}$ and $1095 \mathrm{~K}$, respectively (see Table 1). This chemical shift can be explained by a change of the bonding between the silica film and the Ru substrate, as already discussed in Ref. [33]. Whereas at lower oxidation temperature the distance of the silica film to the Ru substrate is small, the silica-Ru distance is slightly larger at higher temperature due to oxygen adsorption at the interface. This is revealed in the $O 1 \mathrm{~s} \mathrm{spectra} \mathrm{(Fig.} \mathrm{7b).} \mathrm{Here,} \mathrm{the} \mathrm{strongest}$ contribution is due to the oxygen bound to $\mathrm{Si}$ at $\mathrm{E}_{\mathrm{BE}}=532.1 \mathrm{eV}$, whereas the oxygen bound to $\mathrm{Ru}$ has a binding energy of $E_{B E}=529.5 \mathrm{eV}$. At a low oxidation temperature of $995 \mathrm{~K}$, the contribution of the RuO peak is small; however, its intensity increases with oxidation temperature. At the same time, the $\mathrm{SiO}_{2}$ peak shifts towards lower binding energy. Oxidation above $1100 \mathrm{~K}$ leads to a loss in the $\mathrm{SiO}_{2}$ intensity and an increase in that of RuO as expected for the de-wetting of the film observed in LEEM (Fig. 5d).
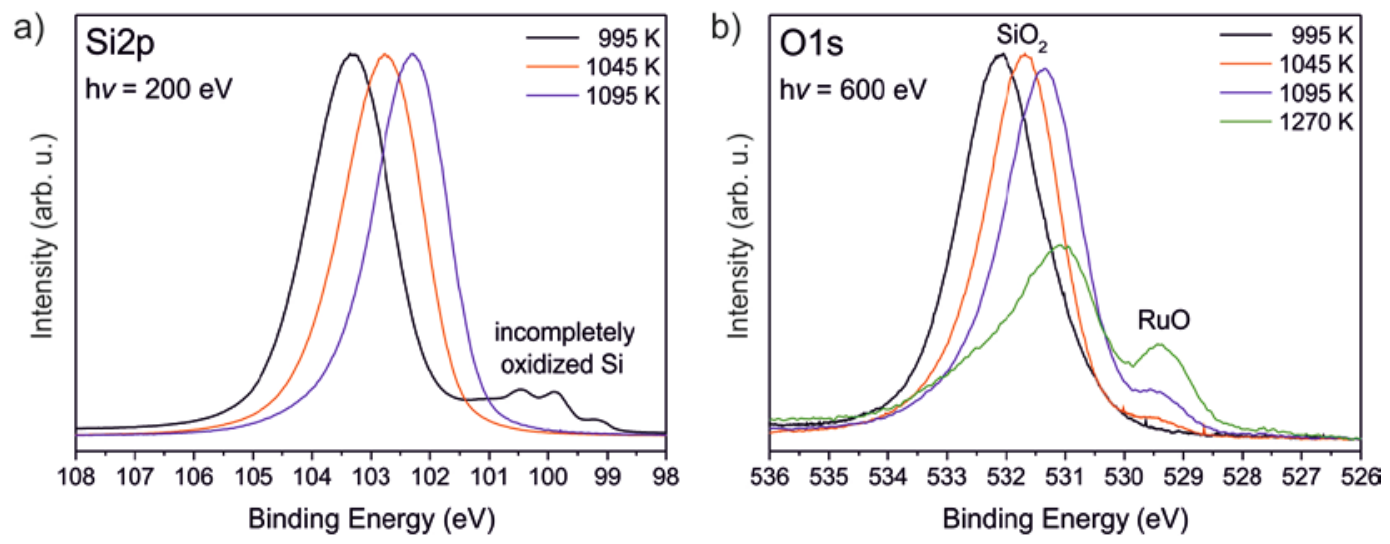

Fig. 7: XPS spectra of Si $2 p$ and $O$ 1s for silica after oxidation at different temperatures. Photon energy is $200 \mathrm{eV}$ (Si 2p) and $600 \mathrm{eV}$ (O 1s), respectively.

\begin{tabular}{|c|c|c|}
\hline Oxidation temperature (K) & BE of Si $\mathbf{2 p} \mathbf{p ( e V )}$ & BE of O 1s (eV) \\
\hline 995 & 103.3 & 532.1 \\
\hline 1045 & 102.7 & 531.7 \\
\hline 1095 & 102.3 & 531.4 \\
\hline 1270 & - & 531.1 \\
\hline
\end{tabular}

Table 1: Binding energy of Si 2p and O 1s XPS peak, depending on oxidation temperature 


\subsection{Film structure at mesoscopic holes}

Depending on preparation conditions the silica film is either well-ordered in a p(2x2) superstructure (socalled "crystalline phase") or disordered ("vitreous phase"). For both phases, we investigated the mesoscopic holes in LEEM, using the structural contrast of dark field LEEM (Fig. 8). For the crystalline phase (top row) the LEED pattern shows the $p(2 \times 2)$ structure with - in this case - additional contributions of the (2x2)R30 $30^{\circ}$ structure (Fig. 8a), whereas the vitreous phase (bottom row) exhibits a diffuse ring in LEED, corresponding to a disordered structure (Fig. 8e). Utilizing two neighbouring $p(2 \times 2)$ diffraction spots for dark field imaging (marked by green and red circles) it is possible to identify the surface area with the corresponding structure. Both, the elongated hole area and the bilayer silica area appear bright and therefore exhibit a $\mathrm{p}(2 \times 2)$ structure. However, the intensity of the hole area is not uniform but shows brighter and darker parts; these are inverted when a neighbouring $p(2 \times 2)$ spot is used. This is due to the threefold symmetry of the $\mathrm{Ru}(0001)-\mathrm{p}(2 \times 2)-30$ surface. Also locally recorded LEED-IV traces within the hole area fit very well to the LEED-IV behaviour known for the pure Ru(0001)$\mathrm{p}(2 \times 2)$-30 surface (not shown). Using this observation as a structural fingerprint, we conclude that the hole exposes an oxygen covered $\mathrm{Ru}(0001)$ substrate surface. Furthermore, utilizing the $(2 \times 2) \mathrm{R} 30^{\circ}$ spot for dark field imaging (blue, Fig. $8 \mathrm{c}$ ), the hole area appears dark indicating that the rotated structure does not exist within the hole area. In contrast, the bilayer is uniformly bright, without visible domains. Hence, the rotated $(2 \times 2) R 30^{\circ}$ is present in the entire silica film, additionally to the dominant $\mathrm{p}(2 \times 2)$

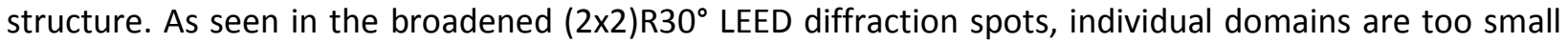
to be resolved with the instrumental resolution of about $13 \mathrm{~nm}$ in these experiments.

The LEED pattern of the vitreous phase (Fig. 8e) consists of a ring around the (00) spot at a distance corresponding to that of the $\mathrm{p}(2 \times 2)$ structure. The LEEM images (Fig. 8f-h) show a hole in the vitreous silica layer using the intensity at the positions of the $\mathrm{p}(2 \times 2)$ and the $(2 \times 2) R 30^{\circ}$ LEED spots (see markings in Fig. 8e) and the (00) spot. Again, inside the hole, the Ru(0001)-p(2x2)-30 surface is visible, as confirmed by the threefold symmetry and the local LEED-IV fingerprint. The area surrounding the hole appears bright in both dark field images ( $f$ and $g$ ), because of the ring intensity at the LEED spot position. However, neither $\mathrm{p}(2 \times 2)$ nor $(2 \times 2) R 30^{\circ}$ structures exist in the vitreous bilayer silica, as local LEED proves

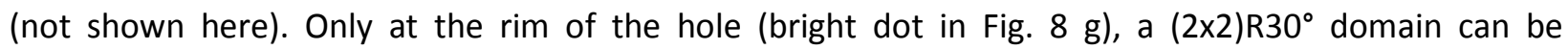
identified by local LEED, indicating that the hole might locally stabilize some residual order in the silica film. 
a)

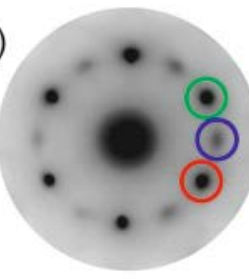

e)

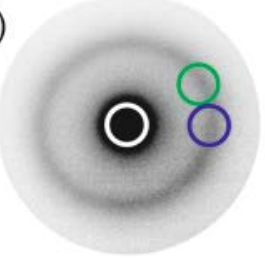

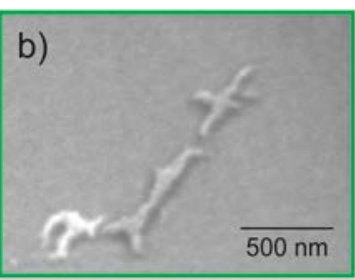

f)

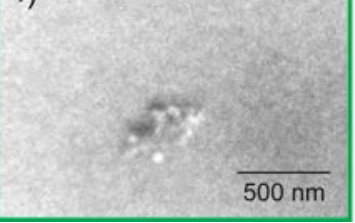

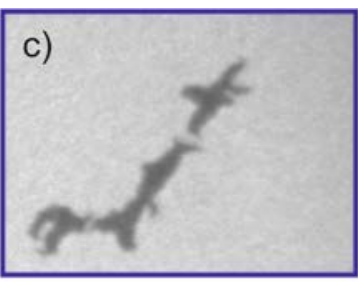

g)

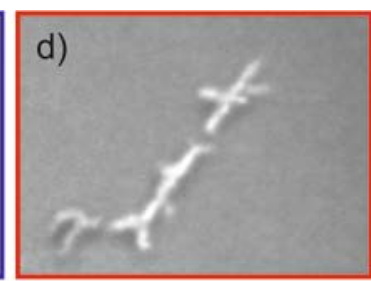

h)
(00)

Fig. 8: Mesoscopic holes in crystalline (top row) and vitreous (bottom) films. LEED pattern (a, e) and bright and dark field LEEM using $\mathrm{p}(2 \times 2)$ (marked in green and red) and ( $2 \times 2) \mathrm{R} 30^{\circ}$ spots (marked in blue) as indicated in the LEED pattern. $E_{\text {kin }}=23 \mathrm{eV}$ in all images.

\section{Discussion}

The experiments concerning Si deposition in an oxygen atmosphere, presented in this work, (section 3.2) yield three main results: (1) temperature dependent nucleation density, (2) dendrite shape of growing silica islands, and (3) reduced reactive sticking coefficient at higher temperature.

From the temperature dependence of the island density an apparent activation energy of $E_{a}=1.65 \mathrm{eV}$ may be derived, which is clearly higher than observed for the nucleation process in metal epitaxy.[34] The measured activation energy is connected with both the binding energy $E_{i}$ of the critical cluster and its diffusion energy $E_{d}$. [35] Since (i) the energy of a covalent bond is a few times higher than that of a metallic bond and (ii) the ring structure of silica might demand a complete ring as a stable cluster (i.e. 6 Si atoms compared to 2 to 3 metal atoms typically needed for a stable metallic nucleus), the high value of $E_{i}$ may cause the high value of $E_{a}$.

The dendritic shape of the growing islands indicates a limited ability for the $\mathrm{Si}$ or $\mathrm{SiO}_{2}$ particles to diffuse along the border of the island (Si sticks nearby the place where it lands at the rim of the silica island). This might also be explained by the strong bonding of $\mathrm{SiO}_{2}$ at the border of already present silica island.

Finally, the reduced sticking coefficient at growth temperatures above $1000 \mathrm{~K}$ is consistent with the observation of de-wetting of silica films at oxidations temperatures above $1200 \mathrm{~K}$ (section 3.3). In the case of oxidation at elevated temperature, the de-wetting and the concomitant desorption occurs at higher temperature. The reason may be that a complete layer is close-packed and is thus particularly

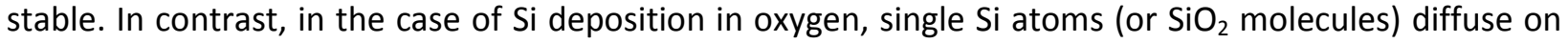
the support and are therefore less strongly bound and less stable. This will lead to desorption at lower temperature. 
On the basis of the present spectro-microscopic results, we identify an optimum oxidation temperature range between $1020 \mathrm{~K}$ and $1180 \mathrm{~K}$ for the "standard recipe". Below this temperature, the film is incompletely oxidized, but closed. On the contrary, above $1200 \mathrm{~K}$, the film is completely oxidized but dewets. Only in the narrow temperature, range around $1100 \mathrm{~K}$ the film is both completely oxidized and closed except for some unavoidable holes.

As shown for the crystalline and for the vitreous phases, we were able to create mesoscopic holes in the film by annealing, which opens the film down to the 30-(2x2) covered $\mathrm{Ru}(0001)$ substrate. These holes exhibit the potential to intercalate reactive gases between the silica film and the Ru support.

\section{Conclusion}

This study has shown that it is possible to grow large scale silica films on Ru(0001) and analyze structure and morphology on a mesoscopic scale. This provides valuable input for the use of ensemble averaging techniques to study properties of such films, including the diffusion of molecules through the film and at the interface between the silica film and the Ru metal surface.

\section{Acknowledgements}

We thank the BESSY II crew for their technical support and the Helmholtz-Center Berlin for Material and Energy for the allocation of synchrotron radiation beamtime. We gratefully acknowledge the financial support by the Federal German Ministry of Education and Science (BMBF) under Contract no. 05KS4WWB/4 and the Deutsche Forschungsgemeinschaft through CRC 1109, as well as by the Fonds der Chemischen Industrie. 


\section{References:}

[1] H.-R. Wenk, A. Bulakh, Minerals. Their Constitution and Origin, first ed., Cambridge University Press, Cambridge, 2004.

[2] T. Schröder, M. Adelt, B. Richter, M. Naschitzki, M. Bäumer, H.-J. Freund, Epitaxial Growth of $\mathrm{SiO}_{2}$ on Mo(112), Surf. Rev. Lett., 7 (2000) 7-14.

[3] T. Schröder, M. Adelt, B. Richter, M. Naschitzki, M. Bäumer, H.-J. Freund, Growth and Well-ordered Silicon Dioxide Films on Mo(112), Microelectron. Reliability, 40 (2000) 841-844.

[4] T. Schröder, A. Hammoudeh, M. Pykavy, N. Magg, M. Adelt, M. Bäumer, H.-J. Freund, Single Crystalline Silicon Dioxide Films on Mo(112), Solid-State Electron., 45 (2001) 1471-1478.

[5] T. Schröder, J.B. Giorgi, M. Bäumer, H.-J. Freund, Morphological and electronic properties of ultrathin crystalline silica epilayers on a Mo(112) substrate, Phys. Rev. B, 66 (2002) 165422.

[6] J. Weissenrieder, S. Kaya, J.-L. Lu, H.-J. Gao, S. Shaikhutdinov, H.-J. Freund, M. Sierka, T.K. Todorova, J. Sauer, Atomic Structure of a Thin Silica Film on a Mo(112) Substrate: A Two-Dimensional Network of $\mathrm{SiO}_{4}$ Tetrahedra, Phys. Rev. Lett., 95 (2005) 076103.

[7] L. Giordano, D. Ricci, G. Pacchioni, P. Ugliengo, Structure and vibrational spectra of crystalline $\mathrm{SiO}_{2}$ ultra-thin films on Mo(112), Surf. Sci., 584 (2005) 225-236.

[8] D. Löffler, J.J. Uhlrich, M. Baron, B. Yang, X. Yu, L. Lichtenstein, L. Heinke, C. Büchner, M. Heyde, S. Shaikhutdinov, H.-J. Freund, R. Włodarczyk, M. Sierka, J. Sauer, Growth and Structure of Crystalline Silica Sheet on Ru(0001), Phys. Rev. Lett., 105 (2010) 146104.

[9] L. Lichtenstein, C. Büchner, B. Yang, S. Shaikhutdinov, M. Heyde, M. Sierka, R. Włodarczyk, J. Sauer, H.-J. Freund, The Atomic Structure of a Metal-Supported Vitreous Thin Silica Film, Angew. Chem. Int. Ed., 51 (2012) 404-407.

[10] R. Włodarczyk, J. Sauer, X. Yu, J.A. Boscoboinik, B. Yang, S. Shaikhutdinov, H.-J. Freund, Atomic Structure of an Ultrathin Fe-Silicate Film Grown on a Metal: A Monolayer of Clay?, J. Amer. Chem. Soc., 135 (2013) 19222-19228.

[11] M. Heyde, S. Shaikhutdinov, H.-J. Freund, Two-dimensional silica: Crystalline and vitreous, Chem. Phys. Lett., 550 (2012) 1-7.

[12] S. Shaikhutdinov, H.-J. Freund, Ultrathin Silica Films on Metals: The Long and Winding Road to Understanding the Atomic Structure, Adv. Mater., 25 (2013) 49-67.

[13] C. Büchner, L. Lichtenstein, X. Yu, J.A. Boscoboinik, B. Yang, W.E. Kaden, M. Heyde, S.K. Shaikhutdinov, R. Włodarczyk, M. Sierka, J. Sauer, H.-J. Freund, Ultrathin Silica Films: The Atomic Structure of Two-Dimensional Crystals and Glasses, Chem. Eur. J., 20 (2014) 9176-9183.

[14] W.H. Zachariasen, The atomic arrangement in glass, J. Am. Chem. Soc., 54 (1932) 3841-3851.

[15] P.Y. Huang, S. Kurasch, A. Srivastava, V. Skakalova, J. Kotakoski, A.V. Krasheninnikov, R. Hovden, Q. Mao, J.C. Meyer, J. Smet, D.A. Muller, U. Kaiser, Direct Imaging of a Two-Dimensional Silica Glass on Graphene, Nano Lett., 12 (2012) 1081-1086.

[16] P.Y. Huang, S. Kurasch, J.S. Alden, A. Shekhawat, A.A. Alemi, P.L. McEuen, J.P. Sethna, U. Kaiser, D.A. Muller, Imaging Atomic Rearrangements in Two-Dimensional Silica Glass: Watching Silica's Dance, Science, 342 (2013) 224-227.

[17] E.I. Altman, J. Götzen, N. Samudrala, U.D. Schwarz, Growth and Characterization of Crystalline Silica Films on Pd(100), J. Phys. Chem. C, 117 (2013) 26144-26155.

[18] X. Yu, B. Yang, J.A. Boscoboinik, S. Shaikhutdinov, H.-J. Freund, Support effects on the atomic structure of ultrathin silica films on metals, Appl. Phys. Lett., 100 (2012) 151608.

[19] J. Weitkamp, Zeolites and catalysis, Solid State lonics, 131 (2000) 175-188.

[20] H. Zhang, Q. Fu, Y. Cui, D. Tan, X. Bao, Growth Mechanism of Graphene on Ru(0001) and $\mathrm{O}_{2}$ Adsorption on the Graphene/Ru(0001) Surface, J. Phys. Chem. C, 113 (2009) 8296-8301. 
[21] Y. Yao, Q. Fu, Y.Y. Zhang, X. Weng, H. Li, M. Chen, L. Jin, A. Dong, R. Mu, P. Jiang, L. Liu, H. Bluhm, Z. Liu, S.B. Zhang, X. Bao, Graphene cover-promoted metal-catalyzed reactions, Proc. Natl. Acad. Sci. U.S.A., 111 (2014) 17023-17028.

[22] L. Jin, Q. Fu, H. Zhang, R. Mu, Y. Zhang, D. Tan, X. Bao, Tailoring the Growth of Graphene on $\mathrm{Ru}(0001)$ via Engineering of the Substrate Surface, J. Phys. Chem. C, 116 (2012) 2988-2993.

[23] L. Jin, Q. Fu, Y. Yang, X. Bao, A comparative study of intercalation mechanism at graphene/Ru(0001) interface, Surf. Sci., 617 (2013) 81-86.

[24] Y. Zhang, Q. Fu, Y. Cui, R. Mu, L. Jin, X. Bao, Enhanced reactivity of graphene wrinkles and their function as nanosized gas inlets for reactions under graphene, Phys. Chem. Chem. Phys., 15 (2013) 19042-19048.

[25] E. Emmez, B. Yang, S. Shaikhutdinov, H.-J. Freund, Permeation of a Single-Layer $\mathrm{SiO}_{2} \mathrm{Membrane}$ and Chemistry in Confined Space, J. Phys. Chem. C, 118 (2014) 29034-29042.

[26] R. Wichtendahl, R. Fink, H. Kuhlenbeck, D. Preikszas, H. Rose, R. Spehr, P. Hartel, W. Engel, R. Schlögl, H.-J. Freund, A.M. Bradshaw, G. Lilienkamp, T. Schmidt, E. Bauer, G. Benner, E. Umbach, SMART: An Aberration-Corrected XPEEM/LEEM with Energy Filter, Surf. Rev. Lett., 05 (1998) 1249-1256.

[27] T. Schmidt, U. Groh, R. Fink, E. Umbach, O. Schaff, W. Engel, B. Richter, H. Kuhlenbeck, R. Schlögl, H.-J. Freund, A.M. Bradshaw, D. Preikszas, P. Hartel, R. Spehr, H. Rose, G. Lilienkamp, E. Bauer, G. Benner, XPEEM with energy-filtering: advantages and first results from the SMART project, Surf. Rev. Lett., 9 (2002) 223-232.

[28] T. Schmidt, H. Marchetto, P.L. Lévesque, U. Groh, F. Maier, D. Preikszas, P. Hartel, R. Spehr, G. Lilienkamp, W. Engel, R. Fink, E. Bauer, H. Rose, E. Umbach, H.-J. Freund, Double aberration correction in a low energy electron microscope, Ultramicroscopy, 110 (2010) 1358-1361.

[29] T. Schmidt, A. Sala, H. Marchetto, E. Umbach, H.J. Freund, First experimental proof for aberration correction in XPEEM: Resolution, transmission enhancement, and limitation by space charge effects, Ultramicroscopy, 126 (2013) 23-32.

[30] A. Sala, H. Marchetto, Z.H. Qin, S. Shaikhutdinov, T. Schmidt, H.-J. Freund, Defects and inhomogeneities in $\mathrm{Fe}_{3} \mathrm{O}_{4}(111)$ thin film growth on Pt(111), Phys. Rev. B, 86 (2012) 155430.

[31] W. Braun, H. Kuhlenbeck, Chemical structure of ultrathin thermally grown oxides on a Si(100)-wafer using core level photoemission, Surf. Sci. , 180 (1987) 279-288.

[32] C.D. Wagner, J.F. Moulder, W.F. Stickle, P.E. Sobol, K.D. Bomben, Handbook of X-ray Photoelectron Spectroscopy: A Reference Book of Standard Spectra for Identification and Interpretation of XPS Data, Physical Electronics Division, Perkin-Elmer Corporation, 1979.

[33] R. Włodarczyk, M. Sierka, J. Sauer, D. Löffler, J.J. Uhlrich, X. Yu, B. Yang, I.M.N. Groot, S. Shaikhutdinov, H.-J. Freund, Tuning the electronic structure of ultrathin crystalline silica films on Ru(0001), Phys. Rev. B, 85 (2012) 085403.

[34] T. Schmidt, E. Bauer, Interfactant-mediated quasi-Frank-van der Merwe growth of Pb on Si(111), Phys. Rev. B, 62 (2000) 15815-15825.

[35] J.A. Venables, G.D.T. Spiller, M. Hanbücken, Nucleation and growth of thin films, Rep. Prog. Phys., 47 (1984) 399-459. 